\title{
Die IDS-Trends: Digitalisierung und Hygiene
}

\author{
Vier Fragen an Timo Kampf, Direktor Marketing Equipment bei Henry Schein Dental Deutschland
}

djz: Herr Kampf, was waren die Topthemen der IDS 2015?

Timo Kampf: Das übergreifende Thema war die Digitalisierung des prothetischen Workflows zwischen Praxis und Labor. Viele reden nur vom digitalen Abdruck; es geht aber auch darum, alle therapierelevanten Schritte abzudecken - beispielsweise 3D-Röntgen, Design-/Planungssoftware oder 3D-Printing. Auch das Fräsen moderner Hochleistungsmaterialien und neue Software-Lösungen waren ein Thema.

Ein weiterer Trend: Hygiene. Hier dreht sich alles um die RKIAnforderungen an die Praxis und wie diese effizient und nachhaltig implementiert werden können.

djz: Muss man als Zahnarzt heute digital denken?

Kampf: Ohne digitale Prozesse lässt sich die heute geforderte Präzision nicht mehr liefern. Wenn ich die Chance habe, über die gesamte Prozesskette hinweg digital zu arbeiten, erhalte ich eine durchgängige Präzision ohne manuelle Übertragungsfehler. Dazu kommt noch die einfache und schnelle Verfügbarkeit digitaler Daten und der schnelle Austausch mit Kollegen oder dem Labor.

djz: Was raten Sie Zahnärzten, die sich niederlassen möchten? Kampf: Die Digitalisierung bietet viele Chancen auf Effizienz und Sicherheit, aber auch Chancen, sich abzugrenzen. Man muss nicht zwingend mit einer Design-Praxis starten. Man sollte sich mit seinem Team und seinem Leistungsspektrum so aufstellen,
Timo Kampf //

Direktor Marketing Equipment

Henry Schein Dental Deutschland

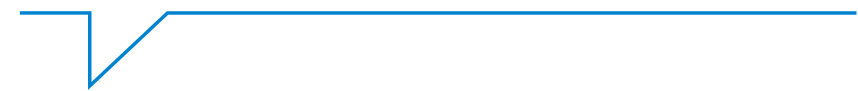

dass man in seinem Umfeld eine gewisse Einzigartigkeit aufbaut und einen Mehrwert für Patienten bietet.

djz: Wie wichtig sind vorhandene Geräte bei der Praxisübernahme? Kampf: Nicht das vorhandene Inventar ist maßgeblich für den Erfolg einer Praxis, sondern andere Faktoren wie die Erreichbarkeit, Servicezeiten oder die Lage. Geräte und Einheiten kann man jederzeit tauschen. Der Zahnarzt sollte sich den Standort genau anschauen und bewerten. Einzel- und Landpraxen sind rückläufig, dabei kann eine Praxisübernahme auf dem Land wirtschaftlich sehr interessant sein. Das Basis-Setup muss jedoch stimmen, damit er direkt starten kann. Später kann er sich auf seinen Schwerpunkt konzentrieren und in modernes Equipment investieren.

\section{Hier steht eine Anzeige.}

